\title{
Preparing astronomical observations and observing with OHP facilities
}

\author{
J. Patris ${ }^{\mathrm{a}}$
}

Université Paul Cézanne, Faculté des Sciences et Techniques, Avenue du Pigonnet, 13090 Aix-en-Provence, France

\begin{abstract}
In addition to the general introduction to observational astronomy given in a past paper (ERCA 07 [13]) some tools are given to the observer. These include the general knowledge of what is going to be observed and of the instruments used. A guideline is given to prepare an observing program and to follow it as efficiently as possible. Finally, some examples are given of observations to be made by ERCA participants at OHP observatory.
\end{abstract}

\section{Introduction}

For some years, ERCA participants, without being specialised astronomers, have had the opportunity of using OHP facilities in astronomy. In a past paper (ERCA 07 [13]), we gave some basics of astronomy and presented the observatory's instruments. In this paper, we aim to give to ERCA participants (or any non-experimented observers) the means of achieving scientific observations. This includes knowing what kind of object is observable with a given telescope, when it is possible to observe it, and how to do it as efficiently as possible.

\section{What shall we observe?}

The first question to address when planning astronomical observations is of course the aim of the observation. The observations are usually part of a scientific or pedagogical project. For a scientific project, the observer will choose a list of precise and similar targets. For a pedagogical project, on the other hand, it is more interesting to observe a wide sample of astronomical objects. We sum up in the following part what kind of astronomical objects can be seen with an optical telescope such as the ones used in OHP.

\subsection{Objects in the sky}

Three very different scales of objects are visible in the sky: objects from our solar system, objects in our galaxy, and extragalactic objects. From the observer's point of view, they are characterised mainly by their luminosity (or magnitude) and their position (or coordinates) in the 2-dimensional night sky.

Extragalactic objects visible from an optical telescope are galaxies, ranging from our neighbourgs the Magellanic Clouds (only visible in the southern hemisphere) and Andromeda galaxy (our Milky

\footnotetext{
a e-mail: julie.patris@univ-cezanne.fr
}

This is an Open Access article distributed under the terms of the Creative Commons Attribution-Noncommercial License 3.0, which permits unrestricted use, distribution, and reproduction in any noncommercial medium, provided the original work is properly cited. 
Way's sister in shape and size), to very remote objects distant of thousands of megaparsecs (1 Mpc is about 3 million light years). Galaxies are disposed homogenously in the sky, and thus are visible in almost every part of the sky, except in the busy zones of our foreground Milky Way. These zones are too crowded with stars and nebulae to offer a good transparency towards extragalactic world. Galaxies are in general very faint and have a very low surface brightness, and thus a very low contrast in imaging: a dark sky, with no moon, is necessary to observe them.

Astronomical objects in our galaxy (the Milky Way) are normally either stars or nebulae. Stars are point like objects, with a very good contrast and usually good brightness. Their visual aspect can be a little disappointing to an enthusiastic beginner in astronomy, but their spectra are rich of information, and their colors sometime visible even to the unaided eye. They can be grouped in clusters, such as the remarkable globular clusters, wide objects disposed in a large spherical halo around our galaxy. Nebulae are widespread objects sharing the property of the galaxies of beeing simultaneously beautiful and difficult to observe. Their surface brightness and contrast are generally low, except for a few very well known examples such as Messier objects (see [11] for a good presentation of Messier catalogue of striking astronomical objects).

Astronomical objects in our solar system are the only objects whose movements in the frame of far away stars are significant. Thus, finding them can be more tricky than any other object. A useful tool to compute their position at a given time is given by IMCCE (Institut de Mécanique céleste et de calcul des éphémérides [6]). The moon and some of the planets are a wonderful sight through even a little telescope, but are usually too bright to be imaged with a professional telescope and a CCD captor. Catching an asteroid is possible with a good pointing system, as well as a comet if a visible one is announced. For a list of small bodies observable one given night, see the Jet Propulsion Laboratory of the NASA [8].

Before an observing run, it is of course necessary to choose the targets. Messier catalogue [11] can be a help for beginners wishing to discover very beautiful objects. Professional astronomers and students will of course have a list of interesting objects, in their field of research. In any case, once the objects are identified two data shall be checked: their position on the sky during the planned observing night, and their brightness.

\subsection{Position}

The position of an identified object is given in catalogues. A very good on-line catalogue is given by the Observatoire Astronomique de Strasbourg with Simbad astronomical database [3].

The coordinates given by any catalogue are normally in the equatorial system: both right ascension (R.A.) and declination (Dec) are given (see Fig. 1 or [13] for more details). This system is referenced to the vernal point $\gamma$, which is fixed within the frame of faraway stars (not including the precession of the equinoxes, which only affects coordinates on long periods). Thus, all astronomical objects (except in the solar system, see 2.1) have fixed equatorial coordinates to a good approximation.

Another system is necessary to find an object for an astronomer at a given place and a given moment: the position in the Hour Angle system of coordinates (H.A. and Dec), as well as the Local Sidereal Time (ST) at the moment of the observations.

Conversion from local time to sidereal time can be obtained on a number of websites (see [16] to compute sidereal time, and [1] for a detailed explanation of sidereal time).

Hour Angle can then be computed by the following formulae:

$$
H A=S T-R A
$$

Hour angle is linked to the observer, since it is equal to zero on the local meridian line (the line in the sky joining the celestial poles and passing by the observer's zenith). An HA close to 0 is a good sign of visibility of an object in the sky.

In Fig. 2, the whole sky is seen from the point of view of an observer in northern hemisphere, at a latitude close to OHP's (around $43^{\circ} \mathrm{N}$ ). The declination of the points on the celestial equator is $0^{\circ}$, the declination of the Polar Star is $+90^{\circ}$, and the declination of the South celestial pole would be $-90^{\circ}$ 


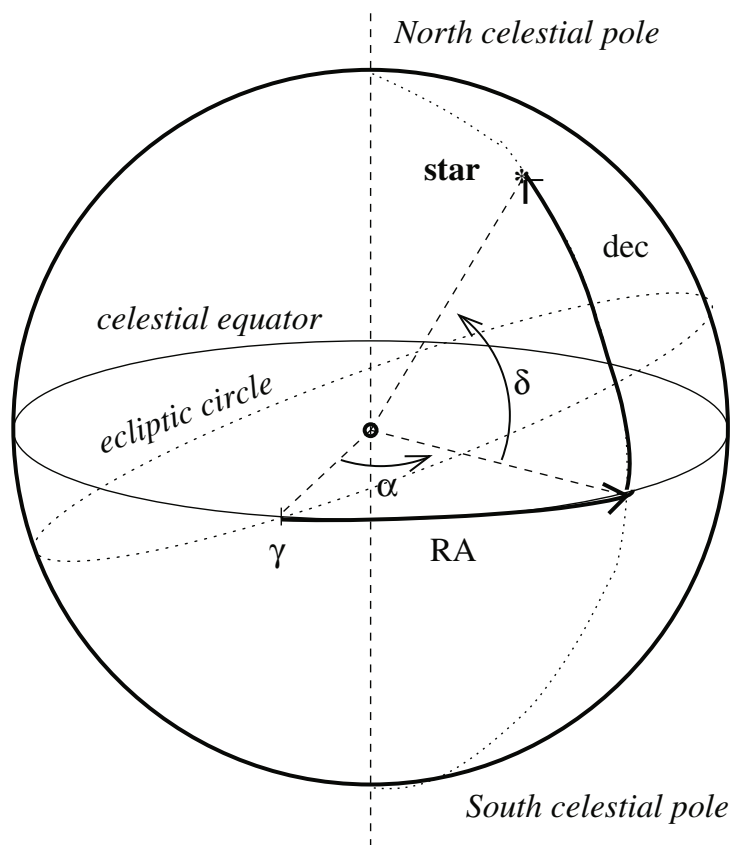

Fig. 1. Definition of equatorial coordinates system on the sky. Observer is at the center of the sphere, which has an arbitrary radius of $\infty$.

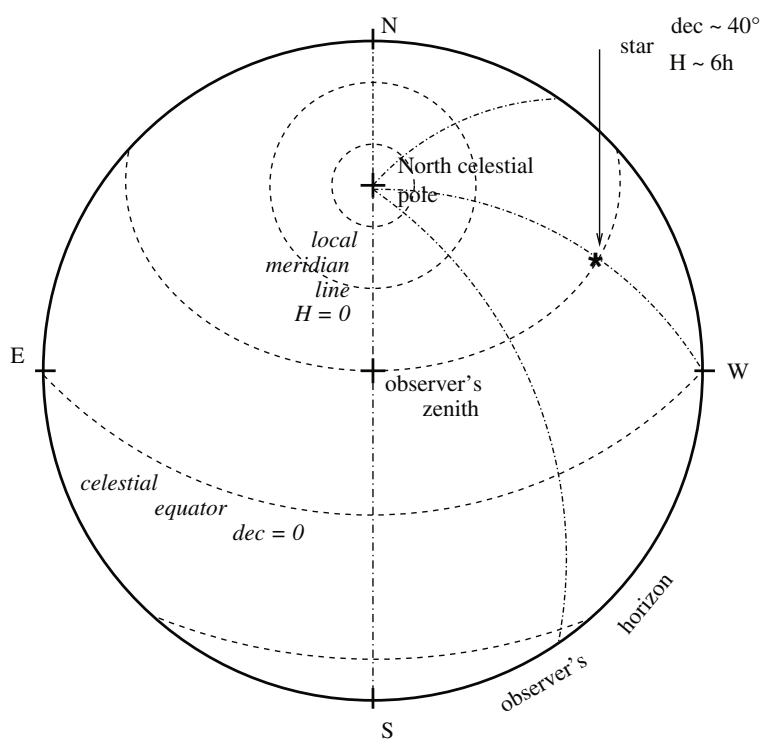

Fig. 2. Coordinates in the sky seen by an observer in the northern hemisphere. In the circle's center is the observer's zenith, and the four geographical cardinal points are plotted on the observer's horizon.

(not visible in northern sky). The points on the local meridian line are at $H=0$, geographical east is at dec $=0^{\circ}$ and $H=-6 \mathrm{~h}$, west beeing at dec $=0^{\circ}$ and $H=+6 \mathrm{~h}$.

With some telescopes, one only needs equatorial coordinates: the telescope will do the conversion and the pointing of the target (this is the case for the big telescopes at OHP). With some telescopes however, like the $0.8 \mathrm{~m}$ instrument at $\mathrm{OHP}$, the observer needs to compute the Hour Angle before observing. 
In any case, knowing the approximate position of the target in the sky is mandatory: to know beforehand whether it will be visible or not (this question will be addressed in section 4), or even to know if the telescope is roughly pointing in the right direction (an initialisation error can happen and the astronomer should be able to detect it as soon as possible!!).

\subsection{Brightness}

The brightness of an object is the flux of light emitted by the object and received in a 1-square-meter section of Earth. It depends mainly on three parameters:

- the object's intrinsic properties and total luminosity $L$,

- the distance $d$ between the object and the observer (the spatial dilution leads to a flux of $F=$ $\left.L /\left(4 \pi d^{2}\right)\right)$

- the interstellar medium, because of the flux absorbed along the line of sight by dust and other interstellar particles.

The resulting brighness of the astronomical object will also depend on wavelength. We usually prefer using an equivalent to brightness in logaritmic scale call "magnitude", and defined by:

$$
m=-2,5 \log \left(F / F_{0}\right)
$$

where:

- $m$ is the magnitude (or apparent magnitude) in a given zone of wavelenght (for instance $m_{V}$ would be visual magnitude, roughly from $500 \mathrm{~nm}$ to $600 \mathrm{~nm}$, see Fig. 5 for the bandwidth of $V$ filter),

$-F$ is the object's total flux in this waveband,

$-F_{0}$ is a flux of reference in this waveband (for instance, the brightness of Vega, see [5] for up to date information on the system of flux standards in astrophysics).

For a point-like object (mainly for stars, but also some galaxies), the contrast on the image, and thus the object's visibility, is directly linked to the object's magnitude. The observer will define the minimum signal/noise ratio acceptable for his purpose. Then, the blackness of the sky, the telescope's aperture, and the captor's efficiency will set how long an exposure will be necessary for a given magnitude. As an example, a signal to noise ratio of 30 was achieved by Roger Cayrel on Elodie instrument, mounted on OHP $193 \mathrm{~cm}$ telescope, while taking the spectrum of a star of magnitude 12 [10].

For an extended object, the magnitude per square angle must be given. It has to be brighter than the background black sky, which has the following typical values at OHP in visible light: $m_{V \text { sky }}=$ $20,7 \mathrm{mag} / \operatorname{arcsec}^{2}$.

The size of the field of view is also important in the case of an extended object (galaxy, nebulae). For instance, the great Andromeda galaxy, spanning a few degrees in the sky, will be impossible to observe with the $0.8 \mathrm{~m}$ OHP telescope, which has a field of view of about $15 \mathrm{arcmin}$.

\subsection{The night sky}

The night sky quality is the first factor of choice when constructing an observatory. OHP was chosen for one of the best skies in France: low air pollution, few cities around (though Manosque is growing fast and its halo is clearly visible south of the OHP, see [7] for more information about light pollution, and [14] for OHP light pollution), and, above all, a low rate of clouded nights.

In a clouded night, it is clearly impossible to observe in visible light. But even in a very clear night, the observer has to take into account the effect of the atmosphere. These are summarized in the concept of "seeing". The night's seeing is the size of the image of a point-like object in the CCD captor for a well focused given instrument. To measure it, an image of a bright star can be taken at the begining of the observations. The seeing is given by the width of the image (an exact measure is the FWHM, full width at half maximum of the light spot) in arcseconds. Without atmosphere, the angular size of 

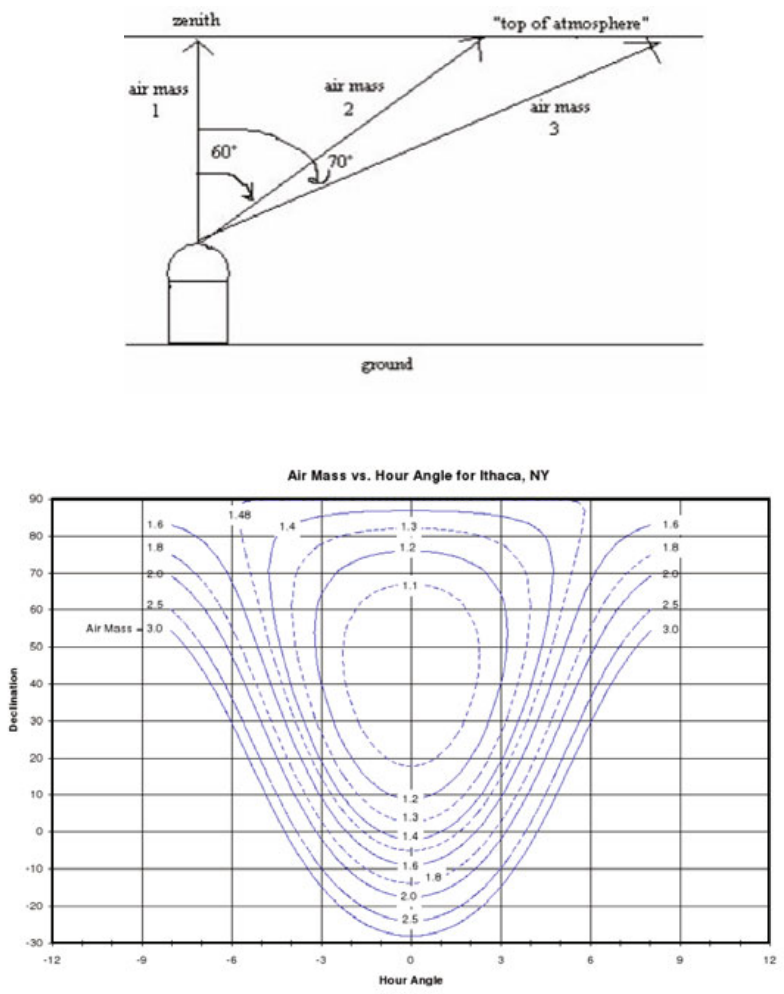

Fig. 3. The airmass a) (top) geometrical definition b) (bottom) airmass for any position in the sky computed for a latitude of $42.5^{\circ}$, close to OHP's latitude. Documents from Cornell College [9].

the spot would be given by the diffraction image of the telescope's aperture (in visible light, roughly 14 arcseconds divided by the aperture in $\mathrm{cm}$, wich means an image of 0.15 arcsecond for $1 \mathrm{~m}$-class telescopes). Because of air movements, the image never reaches this accuracy. The seeing at OHP is limited to a maximum of 2 arcseconds.

The seeing is linked to the instrument's resolution: a binary system can't be separated into two stars if the separation between the stars is less than the seeing.

The best seeing is also linked to the direction observed in the sky: the best observations are made when looking around the observer's zenith. If the altitude of the star is lower, the length of atmosphere crossing the line of sight increases from a simple geometrical effect (see Fig. 3(a)) and the light emitted by the star will be significantly more absorbed and blurred. The proportion of atmosphere crossed by the line of sight is called the "airmass", and is given (at high altitudes above horizon) by:

$$
X=\sec z=\frac{1}{\cos z}
$$

with $z$ the angle between the line of sight and the zenith. Airmass should be as close to 1 as possible. Figure 3(b) shows the air mass as a fonction of the position on the sky, given in Hour Angle coordinates (see part 2.2).

In addition to blurring and absorbing the incident light, atmosphere also emits its proper light. This is a very low signal in the sky (black sky emission of around $20 \mathrm{mag} / \operatorname{arcsec}^{2}$ at OHP) but it can be more important for spectroscopy. The sky intrinsic brighness (pollution excluded) is emitted in narrow pics of wavelength, mainly in the IR light, but also in the green (oxygen recombination line, characteristic of northern lights). Figure 4, taken from [2], gives a typical spectrum of a dark sky emission. 


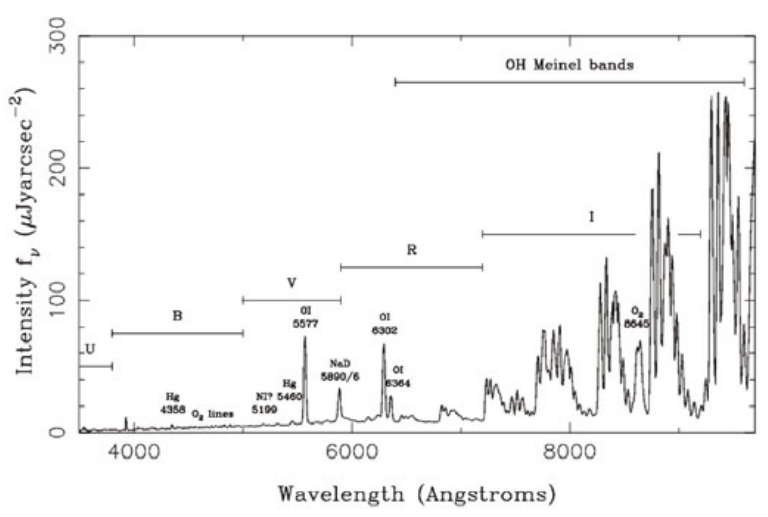

Fig. 4. Spectrum of La Palma night sky, from [2].

\section{What instrument shall we use?}

\subsection{The telescopes}

Three kinds of observation can be done with OHP facilities: visual observation (looking directly throught the telescope), imaging (the telescope is used without ocular lens but with a filter wheel and a CCD captor) or spectroscopy (no eyepiece, but a spectroscope and a CCD camera).

Visual observations are done mainly with the $0.8 \mathrm{~m}$ telescope, wich can be equipped with one of several eyepieces. Detailed information about this telescope are given on OHP website [12]. Imaging is done either with the $0.8 \mathrm{~m}$ telescope or with the $1.2 \mathrm{~m}$ telescope. Both are equipped with a CCD camera and a software for data reduction. Spectroscopy is installed at $1.93 \mathrm{~m}$ and $1.52 \mathrm{~m}$ telescopes.

The choice of the instrument is not always given to the observer, but if it is, the main characteristic of the telescope is its aperture: the bigger the aperture is, the more luminous the observed object will be. Thus, to observe a dim object in a very dark night it will be very important to use a large telescope. On the contrary, to observe a bright object, on a moon-illuminated night, a smaller telescope will probably be quite enough.

\subsection{The setting of the telescope}

Visual observation are almost only used for pedagogical purpose. In this case, the astronomer will try to pick up a set of very different objects. He will usually use first a large field eyepiece (big focal length, e.g. $5 \mathrm{~cm}$ ). This is useful to find the object in the sky, and to learn the pointing system (in the case of $0.8 \mathrm{~m}$ telescope, we have a manual pointing system). Once the object is found and the followup engine is ON, a bigger lens can be put to see more details. However, it must be reminded that the observer will never reach a precision better than the night's seeing (see 2.4), even with a very large lens. Things will appear bigger (except point-like stars ...) but fuzzier.

For imaging, the observer will have to choose the filter used. This can be a broadband filter (see Fig. 5 for a usual set of UV, Blue, Visible, Red and near-IR filters) or a narrowband: narrowband filters centered on $\mathrm{H} \alpha$ emission line (at $656 \mathrm{~nm}, 5 \mathrm{~nm}$ of width) are often used to enhance HII gaz in some images, to track star formation in galaxies or hot gaz in nebulae.

The exposure time will naturally be longer if a thin filter is used. A good idea is generally to take a first comparatively short exposure with a $\mathrm{V}$ or $\mathrm{R}$ filter (depending on the object's known color) and then launch a long exposure (around 20 times longer if the filter is 20 times narrower).

Spectroscopy needs longer exposures in general. The grating has to be chosen by the observer, if possible well in advance, as the changing of the grating during the night will take a long time (all the more because the system has to come back to its temperature of equilibrium before any calibration measurement can be done). Usually, a short exposure will be taken to check the position of the target compared to the spectroscope's entrance slit, and then a long exposure will be launched (an exposure of one hour is a typical value for spectrometry). 


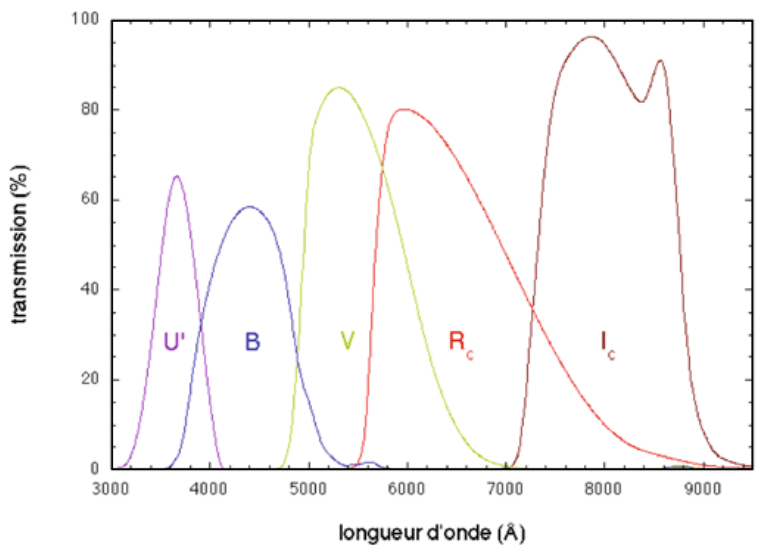

Fig. 5. Wavebands of the filters in use on OHP $1.2 \mathrm{~m}$ telescope. Document S. Ilovaisky [12].

\section{Preparing the night's program}

The first step when preparing observation is to consider the night's conditions. The observer will check first the time of the beginning and the end of the night, and will make sure to meet the person in charge of the telescope well before the observations. Technical information on the instrument is always available on internet: blind zones of the telescope, field of view, filters or gratings, camera specifications ... they have to be read before observing.

The observer will check the value of the Sidereal Time at the beginning of the observing night (using internet calculator [16] for instance). The position of the moon (rising and setting times, phase) is of importance since no faint astronomical target can be observed at full moon...

The general conditions of the observing night known, it is time to choose the objects to observe. Researchers will have their own scientifical goals and know what kind of objects they need. Pedagogues will find a useful number of websites on astronomy:

- IMCCE [6] for the planets' visibility,

- Meudon observatory [11] for Messier objects,

- the Jet Propulsion Laboratory [8] for a list of observable small bodies (asteroids and comets) at any given nights,

- any well-kept amateur astronomer website ...

Once one object (identified by a name and/or a catalogue number) is chosen, the visibility (in terms of brightness and position) has to be checked.

First the brightness of the object must be compared with the possibilities of the telescope. The maximum magnitude (minimum brigthness) observable with a telescope is difficult to define since it depends on the exposure length, and the quality of the night. The exposure time, for a given telescope and a given object, is thus largely determined by experience. Most observatories, however, give "ETCs" (exposure time calculators) to compute the exposure time for a given required signal to noise ratio and a given instrument (see for instance ESO website [4]) .

The position in local coordinates system (Hour Angle and Declination) during the night must be known and compared with a local airmass graphic (Fig. 3). Some skymap softwares (like Stellarium [18]) can be very practical for beginners to check visually the aspect of the sky during the night.

Alternatively, the on-line applet "Staralt" [17] allows to draw a graphic of the altitude above the horizon, and the proximity of the moon, of any object in the sky. Figure 6 give an example of this very useful tool.

The objects will then be chosen, and classified, according to their visibility along the night. It can be a good idea to prepare a "priority 2" program in case the first doesn't fit properly.

Before the observing run, the observer will thus have his list ready, with at least:

- the time in local time, universal time, and sidereal time,

- the name of the object to observe at this moment, 


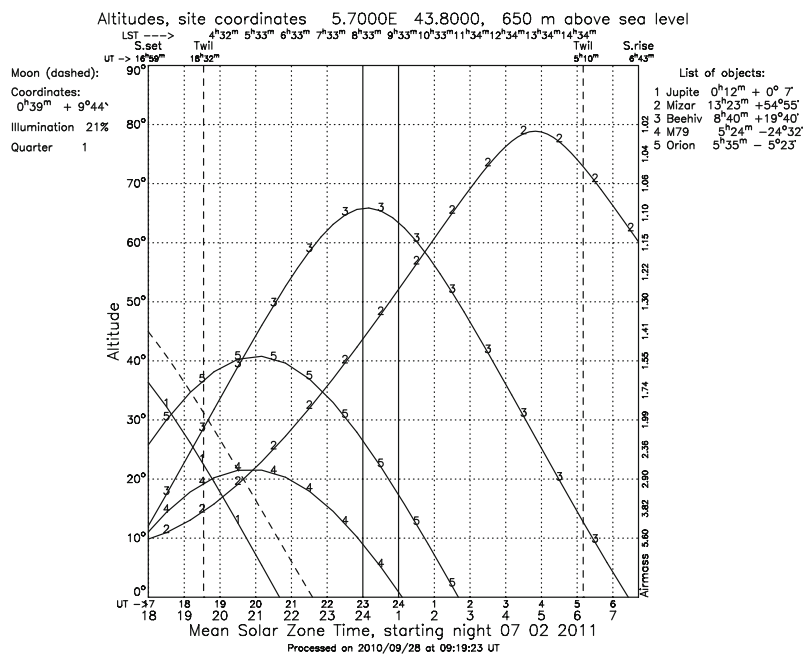

Fig. 6. Altitude of 5 selected objects during the $7^{\text {th }}$ of feb, 2011. Taken from Staralt [17].

- the type of object (star, galaxy, ...)

- the coordinates in equatorial system, precise to the arcmin,

- the coordinates in local system (HA and Dec), and the altitude above the horizon,

- the chosen filter, or the grating,

- the magnitude in the useful waveband.

Even if the coordinates of the object are good, the pointing of the telescope is not often precise to the arcmin (which is the size of the field-of-view), because of mechanical deformations of the tube, due to temperature and position changes. Thus, before any long exposure, it's necessary to take a short exposure to check the position of the target.

However, some objects are so faint they cannot be seen during a short exposure. In this case, it's quite important to prepare an "finding chart". This chart is a picture of the sky taken from a catalogue, with a field of view corresponding to the field of view of the intrument, and centered on the object. The bright stars around the object will help checking whether the pointing is correct.

A finding chart can be drawn around any point of the sky with Simbad software from Strasbourg Observatory [3].

During the observations, apart from science observations, the astronomer will have to take a few other technical exposures:

- the focus,

- the calibration data.

Focus exposure is a special image of one bright point-like object used to check the focus of the instrument. It is done once or twice each night, since the focus can be slightly changed due to temperature and position changes.

Calibration data is necessary for all scientific exposure. It includes "flat fields" at dusk and dawn , dark fields and offsets. These exposures are detailed in [13].

\section{Example}

As an example, let's examine the program of a hypothetical observing run for ERCA participants on February, $7^{\text {th }}$ 2011. Two telescopes are supposed to be booked for them, the $0.8 \mathrm{~m}$ and the $1.2 \mathrm{~m}$ telescopes, till midnight. 
Table 1. Astronomical circumstances for Feb, $7^{\text {th }}, 2011$. See text for references. UT: universal time, LT: local time.

\begin{tabular}{lcc}
\hline event & UT & LT \\
\hline sunset & $16 \mathrm{~h} \mathrm{54 \textrm {min }}$ & $17 \mathrm{~h} \mathrm{54 \textrm {min }}$ \\
end of astronomical twilight & $18 \mathrm{~h} \mathrm{33 \textrm {min }}$ & $19 \mathrm{~h} \mathrm{33 \textrm {min }}$ \\
moonset & $21 \mathrm{~h} \mathrm{34} \mathrm{min}$ & $22 \mathrm{~h} \mathrm{34} \mathrm{min}$ \\
begining of astro. twilight & $5 \mathrm{~h} 09 \mathrm{~min}$ & $6 \mathrm{~h} \mathrm{09min}$ \\
sunrise & $6 \mathrm{~h} \mathrm{48} \mathrm{min}$ & $7 \mathrm{~h} 48 \mathrm{~min}$ \\
\hline
\end{tabular}

\subsection{Definition of the goals}

After reading the instruments' specifications on OHP website [12], we see that the $0.8 \mathrm{~m}$ telescope is equipped with an eye-piece and has to be pointed by hand, whereas the $1.2 \mathrm{~m}$ telescope is equipped with a CCD captor and is handled by a specialist.

We then decide to spend half the time on each telescope, the $0.8 \mathrm{~m}$ telescope being used for a practical learning of sky coordinates and telescope pointing, and the other one to observe a faint object, not visible without a long exposure, and learn the basics of data reduction.

\subsection{The night's astronomical circumstances}

From OHP website [12], we learn the geographical coordinates of the observatory:

- longitude $5^{\circ} 42^{\prime}$ East

- latitude $43^{\circ} 56^{\prime}$ North

- altitude $650 \mathrm{~m}$ above see level.

With this data we can learn the time of the sunset on feb, $7^{\text {th }} 2011$ from a website $[6,19]$ or a software [18] and we get the astronomical circumstances given in table 1.

The observing time will thus last from $6 \mathrm{pm}$ to $12 \mathrm{pm}$ : we can plan almost 3 hours on each telescope. For the first hour, the sky will be rather bright from the sunset and the moon. Thus, it will be quite impossible to observe deep sky objects at the beginning of the night. We decide to use first the $0.8 \mathrm{~m}$ telescope till $9 \mathrm{pm}$ local time, and then to move to the other telescope.

In addition, we compute with IMCCE ephemeris calculator [6] that the sidereal time will be $S T=$ $4,55 \mathrm{~h}=4 \mathrm{~h} 33 \mathrm{~min}$ at $19 \mathrm{~h} \mathrm{UT} \mathrm{(or} 8 \mathrm{pm}$ local time). Sidereal time can be computed any time during the night from this value, knowing that it goes like normal time with an accuracy of more than $1 \%$.

\subsection{Selection of astronomical targets}

For the first part of the observing run, we need bright, beautiful objects. We are lucky to have a very bright object just at the beginning of the night: the moon (magnitude $=-7,7$ ). We'll decide that the first hour and a half will be dedicated to an introduction to the telescope and to an observation of the moon. The pointing will be done without any coordinates, by sight, as a first exercice in telescope handling.

For the next hour and a half, we will prepare five very different objects, thought we probably won't be able to observe more than three. We'd like to pick objects amongst the following types: planet, binary star, open cluster, globular cluster, nebulae.

From a stellarium software [18], from Messier objects [11], and from an Ephemeris calculator (IMCCE, [6]) we choose the following objects:

- planet: Jupiter (magnitude - 1.6),

- binary star: Alcor and Mizar (mag 4 and 2.2) 
Table 2. Observing list for the $0.8 \mathrm{~m}$ telescope on Feb, $7^{\text {th }}$ 2011. $S T=3 \mathrm{~h} 33$ when $U T=18 \mathrm{~h}$, and local time is $L T=U T+1 \mathrm{~h}$.

\begin{tabular}{ccccc}
\hline $\begin{array}{c}\text { Time } \\
\text { (UT) }\end{array}$ & name & $\begin{array}{c}\text { RA } \\
\text { (h min s })\end{array}$ & $\begin{array}{c}\text { dec } \\
\left({ }^{\circ}, ”\right)\end{array}$ & $\begin{array}{c}\text { HA } \\
(\mathrm{h} \mathrm{min})\end{array}$ \\
\hline $\begin{array}{c}17 \mathrm{~h} \\
18 \mathrm{~h} 30\end{array}$ & Moon & & & \\
$18 \mathrm{~h} 45$ & Mizar & $00: 12: 45$ & $+00: 7: 30$ & $3: 20$ \\
$19 \mathrm{~h}$ & Beehive & $08: 40: 55$ & $+54: 55: 25$ & $-94: 05$ \\
$19 \mathrm{~h} 15$ & M79 & $05: 24: 00$ & $-24: 33: 00$ & $-0: 36$ \\
$19 \mathrm{~h} 30$ & Orion & $05: 35: 18$ & $-05: 23: 28$ & $-0: 32$ \\
\hline
\end{tabular}

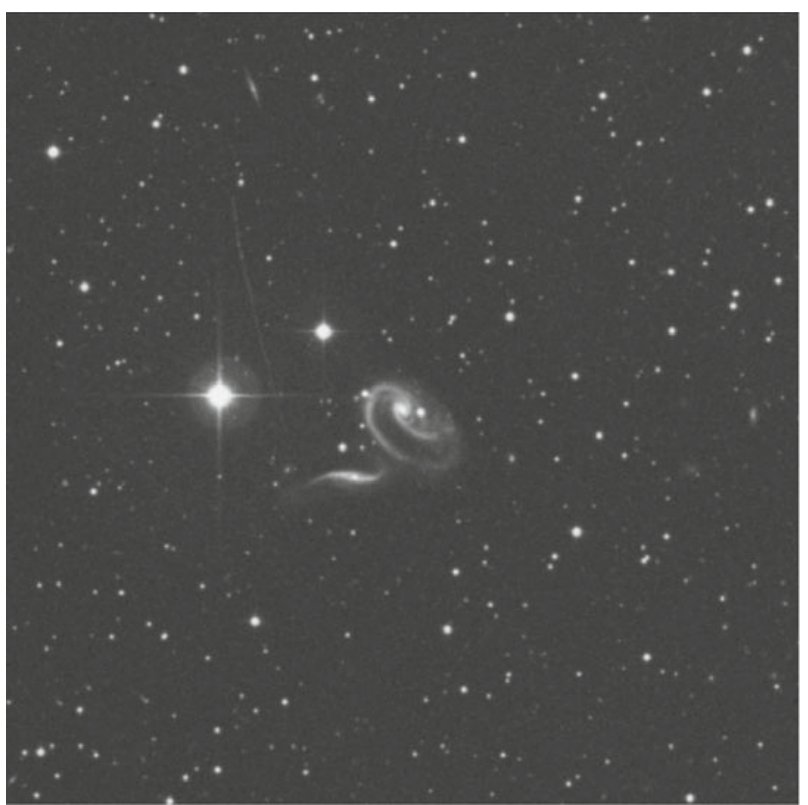

Fig. 7. Finding chart for UGC1810, from POSSII/F/DSS2 all sky survey. Made from Aladin applet [3]. The two bright stars will be used to find the very faint galaxies in the test image (see text).

- open cluster: the Beehive open cluster (mag 3)

- globular cluster: M79 (mag 8.5)

- nebula: Orion Nebula (integrated mag 5).

The "staralt" online software [17] computes the altitude of each object during the night (Fig. 6). We also use the brightness of each object: the Moon of course is the brightest, Jupiter is also very bright and can be observed in the twilight, Alcor and Mizar are two very bright stars and will be seen even if the moon is still high. The Beehive star cluster is also bright, but the two last objects should be observed later, when the night is darker. From all above information, we can plan the observation program given in table 2 .

For the second part of the observing run, on the $1.2 \mathrm{~m}$ telescope, since the moon will set down and we'll have a CCD captor, we chose to observe deep sky objects, such as galaxies, along with a binary star to measure the separation between the stars, and a nebula to check the colors. With the same tools as mentioned above, we construct the end of the following observing list:

- binary: Mizar (separation of 14 arcsec, mag 2,2)

- nebula: Orion Nebula (integrated magnitude of 5) 
Table 3. Observing list for the $1.2 \mathrm{~m}$ telescope on Feb, $7^{\text {th }} 2011$.

\begin{tabular}{cccccc}
\hline $\begin{array}{c}\text { Time } \\
(\mathrm{UT})\end{array}$ & name & $\begin{array}{c}\text { RA } \\
(\mathrm{h} \text { min s })\end{array}$ & $\begin{array}{c}\text { dec } \\
\left({ }^{\circ}, ”\right)\end{array}$ & $\begin{array}{c}\text { exp. } \\
(\mathrm{s})\end{array}$ & filter \\
\hline $20 \mathrm{~h}$ & FField & & & & all \\
& Focus & & & & \\
& dark & & & & \\
$20 \mathrm{~h} 30$ & test & $13: 23: 55$ & $+54: 55: 25$ & $3 \mathrm{~s}$ & $\mathrm{R}$ \\
& Mizar & & & $5 \mathrm{~s}$ & $\mathrm{R}$ \\
& Mizar & & & $5 \mathrm{~s}$ & $\mathrm{~V}$ \\
& Mizar & & & $5 \mathrm{~s}$ & $\mathrm{~B}$ \\
$21 \mathrm{~h}$ & test & $05: 35: 18$ & $-05: 23: 28$ & $5 \mathrm{~s}$ & $\mathrm{R}$ \\
& Orion & & & $15 \mathrm{~s}$ & $\mathrm{R}$ \\
& Orion & & & $15 \mathrm{~s}$ & $\mathrm{~B}$ \\
& Orion & & & $15 \mathrm{~s}$ & $\mathrm{~V}$ \\
& Orion & & & $600 \mathrm{~s}$ & $H_{\alpha}$ \\
$21 \mathrm{~h} 30$ & test & $05: 34: 30$ & $22: 01: 00$ & $10 \mathrm{~s}$ & $\mathrm{R}$ \\
& Crab & & & $30 \mathrm{~s}$ & $\mathrm{R}$ \\
& Crab & & & $30 \mathrm{~s}$ & $\mathrm{~B}$ \\
& Crab & & & $30 \mathrm{~s}$ & $\mathrm{~V}$ \\
& Crab & & & $1200 \mathrm{~s}$ & $H_{\alpha}$ \\
$22 \mathrm{~h}$ & test & $02: 22: 33$ & $+42: 20: 53$ & $10 \mathrm{~s}$ & $\mathrm{R}$ \\
& N891 & & & $30 \mathrm{~s}$ & $\mathrm{R}$ \\
& N891 & & & $30 \mathrm{~s}$ & $\mathrm{~B}$ \\
& N891 & & & $30 \mathrm{~s}$ & $\mathrm{~V}$ \\
& N891 & & & $300 \mathrm{~s}$ & $H_{\alpha}$ \\
$22 \mathrm{~h} 30$ & test & $02: 21: 29$ & $+39: 22: 32$ & $10 \mathrm{~s}$ & $\mathrm{R}$ \\
& U1810 & & & $60 \mathrm{~s}$ & $\mathrm{R}$ \\
& U1810 & & & $60 \mathrm{~s}$ & $\mathrm{~B}$ \\
& U1810 & & & $60 \mathrm{~s}$ & $\mathrm{~V}$ \\
\hline
\end{tabular}

- nebula (supernova remnant): Crab Nebula (integrated magnitude 8.4)

- galaxy (edge on): NGC 891 (integrated magnitude of 10)

- galaxy (interacting pair): UGC 1810 (integrated magnitude 13).

For each object, we plan to take first a quick exposure in $\mathrm{R}$ filter to check the position of the telescope, and then an exposure in each filter. The $H_{\alpha}$ filter needs a longer exposure, as explained in part 3.2. The exposure times given are indicative, the real exposure time will depend on the night's seeing and will be decided after measuring the flux in the first short exposure.

The last object is particularly faint: a finding chart will be necessary to check its position. Figure 7 shows a basic finding chart from Aladin previewer [3].

The resulting preparatory list is given in table 3.

In addition to the scientific exposure, we plan a few "dark" and "offset" exposures, along with a focus and some "flat field" images taken on the closed dome of the telescope.

\section{Conclusion}

All the methods presented here are of course an introduction to preparing useful observations. Professional have all their receipes and habits, but we hope that this introduction will help some beginners in observational astronomy, as well as amateurs.

More details to prepare an observing run can be read on the observatories' websites, such as scientific details on the telescopes or general guides (see the Montreal university's [15]). 
In addition to internet information, specialised on-site staffs are at the observatory to help and advise the astronomers. Meet them well in advance to make sure of the necessary knowledge of the place.

We wish to all long and fruitful observing nights !

Thanks to Alain Sarkissian for the idea of this paper, and to all OHP staff for their constant help at the observatory. Thanks to Sandrine for a fruitful check of my english spelling, and to Luc for his remarks.

\section{References}

1. Aoki S., Guinot B., Kaplan G.H., Kinoshita H., McCarthy D.D., Seidelmann P.K.: "The new definition of Universal Time", Astronomy and Astrophysics 105(2) (1982) pp 359-361

2. Chris R. Benn \& Sara L. Ellison, "La Palma Night-Sky Brightness": http://www.ing . iac.es/Astronomy/observing/ conditions/skybr/skybr.html

3. Centre de données astronomiques de Strasbourg, Simbad astronomical database : http:// simbad.u-strasbg.fr/simbad/

4. ESO website: tools for the observers, exposure time calculators: http://www.eso.org/ observing/etc

5. Fraser, Gerald T. et al. "Absolute flux calibrations of stars", Infrared Spaceborne Remote Sensing and Instrumentation XV. Edited by Strojnik-Scholl, Marija. Proceedings of the SPIE, Volume 6678, pp. 66780P (2007)

6. Ephemeris and position of celestial bodies, IMCCE (Institut de Mécanique Céleste et de Calcul des Ephémérides, Paris): http://www . imcce.fr/langues/en/

7. International Dark-Sky Association : http://www. darksky.org/

8. List of observable small bodies (asteroids and comets), Jet Propulsion Laboratory: http://ssd. jpl.nasa.gov/sbwobs.cgi

9. Julia Kamenetzky, Cornell College: www . cornellcollege.edu/physics/courses/phy312

10. "la lettre de l'OHP": http://www .obs-hp.fr/lettre-ohp/lettre-ohp.html

11. Messier catalogue, observatoire de Paris Meudon : http://messier . obspm.fr/

12. Observatoire de Haute Provence: http://www . obs-hp. fr

13. J. Patris and A. Sarkissian "Astronomical observations with OHP telescopes", J. Phys. IV France ERCA 07 (2006) pp 373-390

14. OHP light pollution website: http://www . obs-hp. fr/pollution/rapport/rapollum. shtml

15. René Racine "conduire des observations astronomiques avec efficacité", 1997: http://www. astro.umontreal.ca/omm/guide ${ }_{\text {fr.html }}$

16. US Navy Sidereal time calculator: http://tycho.usno.navy.mil/sidereal.html

17. Staralt (Isaac Newton Group of telescopes, Spain): http: //catserver .ing . iac . es/staralt/

18. Stellarium software: http://www.stellarium.org/

19. US Navy observatory: http://tycho.usno.navy.mil/ 